\title{
ImmunoPET of CD38 with a radiolabeled nanobody: promising for clinical translation
}

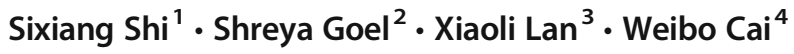 \\ Published online: 3 May 2021 \\ (C) The Author(s), under exclusive licence to Springer-Verlag GmbH Germany, part of Springer Nature 2021
}

Immunotherapy, a therapeutic approach that activates and utilizes a patient's own immune system, has gathered tremendous attention from both patients and researchers, especially after the 2018 Nobel Prize in Physiology or Medicine was awarded jointly to James P. Allison and Tasuku Honjo "for their discovery of cancer therapy by inhibition of negative immune regulation." More than 10,000 studies have been published within the past 2 years for understanding, improving, and clinically translating immunotherapy, based on keyword search in PubMed. For instance, immune checkpoint inhibitors hijacking programmed death-ligand 1 (PD-L1) and its receptor, programmed cell death protein 1 (PD-1), have emerged as the most promising candidates, and several monoclonal antibodies were approved by the Food and Drug Administration (FDA) for treating multiple types of cancer including melanoma, squamous cell lung cancer, renal cell carcinoma, etc. [1]. CD38, a member of the ribosyl cyclase family that is widely expressed on the surface of nonhematopoietic cells and diverse immune cells especially in treatment-resistant tumor microenvironment, has been recently recognized as a novel immune checkpoint protein $[2,3]$. Anti-CD38 immunotherapy can be another promising

This article is part of the Topical Collection on Hematology

Sixiang Shi

sixiang.shi@polyu.edu.hk

$\triangle$ Weibo Cai

wcai@uwhealth.org

1 Department of Health Technology and Informatics, The Hong Kong Polytechnic University, Hung Hom, Hong Kong SAR, China

2 Department of Cancer Systems Imaging, The University of Texas MD Anderson Cancer Center, Houston, TX, USA

3 Department of Nuclear Medicine, Union Hospital, Tongji Medical College, Huazhong University of Science and Technology, Wuhan, China

4 Departments of Radiology and Medical Physics, University of Wisconsin-Madison, Madison, WI, USA treatment for patients who cannot be cured by traditional methods. However, only a small subset of patients really benefits from immunotherapies, while nonresponders suffer from significant side effects [4]. Therefore, effectively distinguishing responders from nonresponders is a critical challenge confronting the clinicians and scientists, and hence, an area of significant research and innovation.

The conventional approaches to distinguishing responders from nonresponders rely heavily on either flow cytometry examination of blood samples or immunohistochemistry of biopsied tumors [5, 6], both of which are invasive, insensitive, and sometimes inaccurate due to the heterogeneous nature of cancer. Whole-body imaging techniques, especially immunopositron emission tomography (immunoPET), can be a critical alternative for guiding patient selection. By administering small doses of radiolabeled antibody-based tracers that bind to the same receptors as that of the therapeutic agents, it is possible to noninvasively, quantitatively, and rapidly evaluate target expression level and hence predict therapeutic efficacy [7]. In addition to patient selection, PET can also be employed to longitudinally monitor the efficacy of immunotherapies, by imaging the activation and/or infiltration of different types of immune cells including T cells, B cells, and macrophages [8]. Thus, PET with suitable tracers can revolutionize treatment planning and monitoring, since repeated biopsy assays on multiple metastatic nodules or deep lesions are neither practical nor accurate.

Among the vast library of radiotracers for immunoPET, nanobody (also named as single-domain antibody, sdAb, or variable domain of a camelid heavy-chain only antibody, VHH), the smallest class of Fc-free antibody fragments, has shown distinct promise [9]. The most important merit of nanobodies for immunoPET is the short blood circulation half-life (usually $<1 \mathrm{~h}$ ), in comparison to intact antibodies (up to several days or weeks) [10], thereby allowing sameday imaging. Zirconium- $89\left({ }^{89} \mathrm{Zr}, t_{1 / 2}=3.3\right.$ days $)$ is typically required to label antibodies to match their long blood circulation $[11,12]$, which could be prone to unwanted bone 
accumulation due to the bone-seeking nature of ${ }^{89} \mathrm{Zr}$. On the other hand, nanobodies allow the use of short-lived isotopes, such as ${ }^{18} \mathrm{~F}\left(t_{1 / 2}=110 \mathrm{~min}\right)$ and ${ }^{68} \mathrm{Ga}\left(t_{1 / 2}=68 \mathrm{~min}\right)$, overcoming the concerns for nonspecific bone accumulation. Importantly, the use of ${ }^{18} \mathrm{~F}$ and ${ }^{68} \mathrm{Ga}$ significantly expands the availability and feasibility of immunoPET, particularly in resource-strapped countries that do not have the capability to produce high-quality ${ }^{89} \mathrm{Zr}$ or ${ }^{64} \mathrm{Cu}$. In addition, the short circulation of nanobodies significantly reduces the background signal in the blood pool and therefore enhances the signal-tobackground contrast $[4,13]$, especially when imaging hematologic cancers, circulating immune cells, or immune organs such as the spleen that are immensely infiltrated with blood. The second merit of nanobody-based radiotracer is its potential renal clearance (due to its low molecular weight $15 \mathrm{kDa})$, unlike whole antibodies $(\sim 150 \mathrm{kDa})$ which are cleared by the hepatic and splenic pathways. Because of renal clearance, nanobodies are especially suitable for imaging abdominal cancers and immune activation that involves spleen and lymph nodes in the abdomen. Thirdly, nanobodies may demonstrate enhanced tissue penetration owing to their smaller size, making them desirable for imaging poorly vascularized or dense tissues/tumors [14].

Building on these advantages, in this issue of European Journal of Nuclear Medicine and Molecular Imaging, Wei, Zhao, Liu, and colleagues reported a ${ }^{68} \mathrm{Ga}$-labeled CD38targeted nanobody, $\left[{ }^{68} \mathrm{Ga}\right] \mathrm{Ga}-\mathrm{NOTA}-\mathrm{Nb} 1053$, to predict the therapeutic outcomes of anti-CD38 immunotherapy in multiple myeloma, a hematological neoplasm of B cell lineage [15]. Good radiochemical yield (> 50\%), excellent radiochemical purity ( $>99 \%)$, and immunoreactivity ( $>95 \%$ ) were achieved, and sharp images of CD38-overpressing tumors were acquired, outperforming the traditional $\left[{ }^{18} \mathrm{~F}\right] \mathrm{FDG}$ PET. $\left[{ }^{68} \mathrm{Ga}\right] \mathrm{Ga}-\mathrm{NOTA}-\mathrm{Nb} 1053$ exhibited excellent specificity, confirmed with the blocking study by pre-injection of daratumumab (therapeutic anti-CD38 antibody). The imaging results correlated well with $\mathrm{CD} 38$ expression levels assessed by cellular and histological analyses, manifesting its potential for early diagnosis and effective patient selection.

Not limited to multiple myeloma, anti-CD38 therapy can be potentially applied to other solid and liquid tumors. CD38 is highly expressed on cancer cells and tumor-associated immune cells, and inhibits $\mathrm{CD} 8^{+} \mathrm{T}$ cell proliferation, antitumor cytokine secretion, and tumor cell killing capability $[2,3]$. Thus, antiCD38 therapies may potentially benefit patients who are resistant to currently approved immune checkpoint inhibitors (such as PD-1/PD-L1). Towards this goal, $\left[{ }^{68} \mathrm{Ga}\right] \mathrm{Ga}$-NOTA$\mathrm{Nb} 1053$-based immunoPET could play an important role in selecting patients who are suitable for anti-CD38 therapy, and predicting and evaluating consequent therapeutic efficacy by longitudinal monitoring of CD38 expression. Therefore, $\left[{ }^{68} \mathrm{Ga}\right] \mathrm{Ga}$-NOTA-Nb1053 immunoPET promises wide applicability and significant potential for clinical translation.
Radiolabeled nanobodies have also been employed in various other studies to noninvasively evaluate the status of the immune system. Ploegh and colleagues from Boston Children's Hospital published a series of articles reporting the applications of radiolabeled anti-CD8 and anti-CD11b nanobodies to accurately track the in vivo biodistribution of $\mathrm{CD}^{+} \mathrm{T}$ cells and $\mathrm{CD} 11 \mathrm{~b}^{+}$myeloid cells [5, 16-18]. The results revealed that the infiltration of $\mathrm{CD}^{+} \mathrm{T}$ cells in colorectal tumors correlated with effective immunotherapy, and homogeneous distribution of $\mathrm{CD} 1 \mathrm{~b}^{+}$myeloid cells in tumors may indicate positive immune responses. The responders exhibited a dominant population of $\mathrm{CD} 11 \mathrm{~b}^{+}$cells with an M1-like signature, while the nonresponders displayed an M2-like transcriptional signature [17]. Imaging of tumor-associated macrophages was also investigated in several other studies, with radiolabeled nanobodies targeting the macrophage mannose receptor (MMR, CD206) [19, 20]. Since recruitment of $\mathrm{MMR}^{+}$tumor-associated macrophages is generally related to poor responsiveness to immunotherapy [21], MMR-targeted imaging may be beneficial in predicting and longitudinally monitoring the response of immunotherapy.

Current research on radiolabeling and immunoPET of nanobodies is almost exclusively preclinical. Many challenges remain and more studies are needed to further improve the nanobodies for clinical translation. Firstly, random-site radiolabeling is the most commonly used labeling method in which the chelators randomly react with amine or other functional groups. This method is suitable for antibodies, because of their large molecular weight and abundant functional groups. However, it could be problematic when labeling nanobodies that have limited number of functional groups. Chelator conjugation with the functional groups within or near the complementarity determining region may interfere with antigen binding. Although one study reported that randomsite radiolabeling did not significantly hamper the binding affinity of nanobodies [6], it is necessary to confirm the biological function of each radiolabeled nanobody before clinical translation, since every type of nanobody has a unique structure. Site-specific radiolabeling may serve as an alternative and superior radiolabeling approach for nanobodies. However, the increased cost of site-specific radiolabeling should also be considered during good manufacturing practices (GMP) production for potential clinical applications.

Secondly, although renal clearance of nanobodies remarkably reduces the radiation burden on the liver and spleen, high kidney uptake should be noted which may lead to potential renal toxicity. However, one study pointed out that the highest dose to the kidneys (up to $90 \mathrm{mGy}$ ) after radiolabeled nanobody administration stayed well below the threshold of 7-8 Gy for potential deterministic effects [20]. Several approaches have been reported to further reduce the renal uptake. In this highlighted study, Wang et al. reported that pretreatment of mice with sodium maleate or fructose 
significantly decreased kidney retention of the PET tracers [15]. Potential health concerns from such strategies that interfere with tubular reabsorption should be considered when applying to patients in the clinical settings. Another study reported the use of PEGylated nanobodies for reduced renal retention without inducing additional side effects [5, 17]. However, such a strategy may decrease the antigen-binding affinity of the nanobodies.

Thirdly, many reported studies on immunoPET imaging were conducted in immunocompromised nude mice bearing xenograft tumors. Higher signal-to-background ratio was achieved in these proof-of-concept studies by avoiding the significant influence from native immune cells. However, the pharmacokinetics of radiolabeled nanobodies in immunodeficient mice may not represent the real in vivo scenario, since the abundant T cells, B cells, and myeloid cells in blood circulation and peripheral tissues may massively interact with nanobodies and lead to significantly different pharmacokinetics and biodistribution. Therefore, immunocompetent mouse models and non-human primate models are recommended for robust testing of nanobody-based immunoPET imaging before clinical investigation.

Immune response is a complex network of interactions involving multiple types of immune cells and cytokines. Even the same lineage of immune cells may behave heterogeneously in different biological processes and pathologies [22]. Therefore, imaging a single biomarker may not be sufficient for robust prediction and evaluation of immunotherapy outcomes. For instance, Rashidian et al. reported that while the intratumoral infiltration of $\mathrm{CD} 11 \mathrm{~b}^{+}$cells in responders positively correlated with the efficacy of anti-PD-1 immunotherapy, $\mathrm{CD} 11 \mathrm{~b}^{+}$cells in nonresponders were believed to stop the entry of $\mathrm{CD}^{+} \mathrm{T}$ cells into the tumor and negatively impacted the therapeutic outcomes [17]. Multi-biomarker imaging may hold the key to precision immunotherapy. Multi-isotope single-photon emission computed tomography (SPECT), which can distinguish signals from different isotopes, can be used to target multiple biomarkers for a better understanding of the complex immune system. In addition, multi-color optical imaging and multispectral optoacoustic tomography can serve as complementary imaging modalities for simultaneously imaging different biomarkers, although they are not as sensitive and quantitative as radionuclide-based imaging. By integrating the concerted efforts from radiologists, oncologists, radiochemists, immunologists, medical physicists, biologists, etc., multimodal molecular imaging approaches may lead the way to potentiate more effective precision immunotherapy at both the preclinical and clinical settings.

As a relatively new cancer marker and immune checkpoint protein, there are still many unknowns about CD38-related biology and potential clinical applications. In this work, radiolabeling of anti-CD38 nanobodies for immunoPET was achieved with excellent radiolabeling quality, imaging contrast, and targeting specificity [15]. Because of the unique advantages of nanobodies and critical role of CD38 in cancer therapy, anti-CD38 nanobody-based immunoPET holds great potential for clinical translation to improve clinical outcomes for cancer patients.

Funding This work received financial support from the Start-Up Fund (P0035391) at The Hong Kong Polytechnic University, the National Natural Science Foundation of China (No. 81630049 and 82030052), the University of Wisconsin - Madison, and the National Institutes of Health (P30CA014520).

\section{Declarations}

Studies with human participants or animals This article does not contain any studies with human participants or animals performed by any of the authors.

Conflict of interest Weibo Cai is a scientific advisor, stockholder, and grantee of Focus-X Therapeutics, Inc. All other authors declare that they have no conflict of interest.

\section{References}

1. Yu JX, Hodge JP, Oliva C, Neftelinov ST, Hubbard-Lucey VM, Tang J. Trends in clinical development for PD-1/PD-L1 inhibitors. Nat Rev Drug Discov. 2020;19:163-4. https://doi.org/10.1038/ d41573-019-00182-w.

2. Chen L, Diao L, Yang Y, Yi X, Rodriguez BL, Li Y, et al. CD38mediated immunosuppression as a mechanism of tumor cell escape from PD-1/PD-L1 blockade. Cancer Discov. 2018;8:1156-75. https://doi.org/10.1158/2159-8290.CD-17-1033.

3. Chen L, Byers LA, Ullrich S, Wistuba II, Qin X-F, Gibbons DL. $\mathrm{CD} 38$ as a novel immune checkpoint and a mechanism of resistance to the blockade of the PD-1/PD-L1 axis. J Clin Oncol. 2017;35:79. https://doi.org/10.1200/JCO.2017.35.7_suppl.79.

4. Lv G, Sun X, Qiu L, Sun Y, Li K, Liu Q, et al. PET imaging of tumor PD-L1 expression with a highly specific nonblocking singledomain antibody. J Nucl Med. 2020;61:117-22. https://doi.org/10. 2967/jnumed.119.226712.

5. Rashidian M, Ingram JR, Dougan M, Dongre A, Whang KA, LeGall C, et al. Predicting the response to CTLA-4 blockade by longitudinal noninvasive monitoring of CD8 T cells. J Exp Med. 2017;214:2243-55. https://doi.org/10.1084/Jem.20161950.

6. Bridoux J, Broos K, Lecocq Q, Debie P, Martin C, Ballet S, et al. Anti-human PD-L1 nanobody for immuno-PET imaging: validation of a conjugation strategy for clinical translation. Biomolecules. 2020;10. https://doi.org/10.3390/biom10101388.

7. Lutje S, Feldmann G, Essler M, Brossart P, Bundschuh RA. Immune checkpoint imaging in oncology: a game changer toward personalized immunotherapy? J Nucl Med. 2020;61:1137-44. https://doi.org/10.2967/jnumed.119.237891.

8. Perrin J, Capitao M, Mougin-Degraef M, Guerard F, FaivreChauvet A, Rbah-Vidal L, et al. Cell tracking in cancer immunotherapy. Front Med. 2020;7:Artn 34. https://doi.org/10.3389/Fmed. 2020.00034.

9. Chakravarty R, Goel S, Cai W. Nanobody: the "magic bullet" for molecular imaging? Theranostics. 2014;4:386-98. https://doi.org/ 10.7150/thno.8006.

10. Keyaerts M, Xavier C, Heemskerk J, Devoogdt N, Everaert H, Ackaert C, et al. Phase I study of 68Ga-HER2-nanobody for PET/ 
CT assessment of HER2 expression in breast carcinoma. J Nucl Med. 2016;57:27-33. https://doi.org/10.2967/jnumed.115.162024.

11. Adams H, van de Garde EM, van Moorsel CH, Vugts DJ, van Dongen GA, Grutters JC, et al. [(89)Zr]Zr-rituximab PET/CT activity in patients with therapy refractory interstitial pneumonitis: a feasibility study. Am J Nucl Med Mol Imaging. 2019;9:296-308.

12. Lau WL, Liang C, Liu H, Singh K, Mukherjee J. Development of zirconium-89 PET for in vivo imaging of alpha-klotho. Am J Nucl Med Mol Imaging. 2020;10:95-105.

13. Xing Y, Chand G, Liu C, Cook GJR, O’Doherty J, Zhao L, et al. Early phase I study of a $(99 \mathrm{~m}) \mathrm{Tc}$-labeled anti-programmed death ligand-1 (PD-L1) single-domain antibody in SPECT/CT assessment of PD-L1 expression in non-small cell lung cancer. J Nucl Med. 2019;60:1213-20. https://doi.org/10.2967/jnumed.118. 224170.

14. D'Huyvetter M, De Vos J, Caveliers V, Vaneycken I, Heemskerk J, Duhoux FP, et al. Phase I trial of (131)I-GMIB-Anti-HER2-VHH1, a new promising candidate for HER2-targeted radionuclide therapy in breast cancer patients. J Nucl Med. 2020. https://doi.org/10.2967/ jnumed.120.255679.

15. Wang C, Chen Y, Hou YN, Liu Q, Zhang D, Zhao H, et al. ImmunoPET imaging of multiple myeloma with $[(68) \mathrm{Ga}] \mathrm{Ga}-$ NOTA-Nb1053. Eur J Nucl Med Mol Imaging. 2021. https://doi. org/10.1007/s00259-021-05218-1.

16. Rashidian M, Keliher EJ, Bilate AM, Duarte JN, Wojtkiewicz GR, Jacobsen JT, et al. Noninvasive imaging of immune responses. Proc Natl Acad Sci U S A. 2015;112:6146-51. https://doi.org/10.1073/ pnas. 1502609112.

17. Rashidian M, LaFleur MW, Verschoor VL, Dongre A, Zhang Y, Nguyen TH, et al. Immuno-PET identifies the myeloid compartment as a key contributor to the outcome of the antitumor response under PD-1 blockade. Proc Natl Acad Sci U S A. 2019;116:16971-80. https://doi.org/10.1073/pnas.1905005116.

18. Van Elssen C, Rashidian M, Vrbanac V, Wucherpfennig KW, Habre ZE, Sticht J, et al. Noninvasive imaging of human immune responses in a human xenograft model of graft-versus-host disease. J Nucl Med. 2017;58:1003-8. https://doi.org/10.2967/jnumed.116. 186007.

19. Blykers A, Schoonooghe S, Xavier C, D’Hoe K, Laoui D, D'Huyvetter M, et al. PET imaging of macrophage mannose receptor-expressing macrophages in tumor stroma using $18 \mathrm{~F}$ radiolabeled camelid single-domain antibody fragments. J Nucl Med. 2015;56:1265-71. https://doi.org/10.2967/jnumed.115. 156828.

20. Xavier C, Blykers A, Laoui D, Bolli E, Vaneyken I, Bridoux J, et al. Clinical translation of $[(68) \mathrm{Ga}] \mathrm{Ga}-\mathrm{NOTA}$-anti-MMR-sdAb for PET/CT imaging of protumorigenic macrophages. Mol Imaging Biol. 2019;21:898-906. https://doi.org/10.1007/s11307-01801302-5.

21. De Palma M, Lewis CE. Macrophage regulation of tumor responses to anticancer therapies. Cancer Cell. 2013;23:277-86. https://doi. org/10.1016/j.ccr.2013.02.013.

22. Satija R, Shalek AK. Heterogeneity in immune responses: from populations to single cells. Trends Immunol. 2014;35:219-29. https://doi.org/10.1016/j.it.2014.03.004.

Publisher's note Springer Nature remains neutral with regard to jurisdictional claims in published maps and institutional affiliations. 